\title{
Does dyslexia present barriers to information literacy in an online environment? A pilot study
}

\author{
Lynne Cole, Andrew MacFarlane, George Buchanan
}

\begin{abstract}
The skills and attributes required to become information literate have not been analysed from the perspective of information users with cognitive disabilities, such as dyslexia, and this research seeks to begin to address this gap in the literature. The initial objective of the research was to collect data showing the online search strategies of dyslexics and non-dyslexics who are in Higher Education, highlighting any areas of difference and difficulty. The results of a pilot study comparing the online search behaviour of seven dyslexic participants with that of seven who were non-dyslexic, are reported here. Participants were adult undergraduates, taken from all three levels of study. Participants were interviewed and their online information searching behaviour was observed through the collection of screen recording diaries over the completion period of one assignment. Within the dyslexic group, difficulties were reported and observed in the areas of keyword creation, use of appropriate tools to refine and expand searches and the evaluation of sources. The dyslexics' group low selfefficacy in many of the skills associated with information literacy was discovered to be notable barrier.
\end{abstract}

\section{Authors}

Lynne Cole is LRC Manager and Lecturer at Norland College.

Email: lynne.cole@city.ac.uk

Andrew MacFarlane is a Reader in the School of Informatics at City University.

George Buchanan is Director of the Centre for Human-Computer Interaction Design at City University.

Received 06 July 2016

Accepted 02 November 2016 


\section{Introduction}

Although $10 \%$ of the population have dyslexia, 4\% severely so (Snowling, 2000; British Dyslexia Association, 2013), only around 3\% of the Higher Education undergraduate student population has a declared specific learning difficulty (Higher Education Statistics Agency, 2015). Within this group, a smaller number will have dyslexia and information literacy has not been analysed from this minority group's information user profile. By investigating whether dyslexia impacts the skills required for information literacy in relation to online searching, focusing on search strategies employed, this report seeks to begin to address this unrepresented area in the literature. It is hoped that wider professional conversations with regards to dyslexia and information literacy and its instruction are instigated.

As a librarian working alongside students with specific learning difficulties in a student support capacity, the motivation for this research came from the recognition that, although there are a plethora of guide books that advise how to adapt teaching and study skills to those with dyslexia, information literacy instruction is yet to be customised in this way. The aim of the research, which is part of a wider doctoral research project, is to consider information literacy from the profile of a dyslexic information user so that recommendations for information literacy instruction can be made.

There are a plethora of definitions and models of information literacy (ACRL, 2015; ALA, 2015; CILIP; 2013; SCONUL; 2011) that feature a wide range of associated skills that must be mastered to become information literate. SCONUL (2011) details a series of understandings and abilities which are required for information literacy. The descriptors included in The Seven Pillars of Information Literacy model developed by SCONUL (2011) are used to evaluate the information literacy of participants in this research. This model was chosen due to the observable and measurable descriptors within each Pillar, which SCONUL affirm to be "skills and competences" and "attitudes and behaviours" (SCONUL, 2011, 3). The Pillars of Identify, Plan, Gather and Evaluate contain information literacy skills that relate to online searching and these are investigated within this study.

Keyword creation and revision are highlighted in Identify and Plan, while Plan also details the skills required to construct search strategies, an ability further developed in Gather. Finally, Evaluate describes the need to be able to "assess the quality of information" (SCONUL, 2011, 9) while at the same time being able to "relate the information found to the original search strategy" (SCONUL, 2011, 9). By investigating the participants understanding and abilities, affective and cognitive challenges will be highlighted and will allow information professionals to consider instructional implications for the teaching of online information literacy skills. 


\section{Background}

\subsection{Cognitive Profile}

In order to research the "skills and competencies" and "attitudes and behaviours" (SCONUL, 2011, 3) that are associated with information literacy from a dyslexic's cognitive profile, the characteristics of a dyslexic information user must first be established. Defining dyslexia can be difficult, as too is diagnosis, because there can be vast differences in individual profiles (Smythe, 2000; Snowling and Stackhouse, 2006; Elliot and Grigorenko, 2014). Nevertheless, there are common difficulties which those with dyslexia will encounter and these manifest themselves in the broad range of skills needed for successful reading, writing and spelling (Rose, 2009).

The symptoms of dyslexia are widely accepted to be caused by a phonological deficit (Stanovic and Siegel, 1994; Snowling, 2000) and common areas of weaknesses are observed in: phonological awareness, verbal memory and verbal processing speed, with these skills being used for diagnosis (Rose, 2009). The British Dyslexia Association (2013) argue that definitions should include visual processing difficulties and that strengths in other areas of development, such as visual thinking skills, should be acknowledged.

Reading and spelling difficulties are persistent throughout life and will impact upon the way dyslexic information users interact with online platforms. Berget, Mulvey and Sandnes $(2016,17)$ note that:

information search requires spelling skills in order to produce accurate and purposeful queries and word recognition skills for exploring results and assessing documents for relevance.

Word recognition involves the use of working and long-term memory. Memory is "the human ability to store, retain, and recall information" (MacKenzie, 2013, 48) and areas in which dyslexic learners commonly show weakness are measurements of verbal working memory and long term memory vocabulary recall. Weaknesses in working memory are due to:

problems with phonological processing [as this] restricts the number of verbal items [dyslexic learners] can retain in memory and has a knock-on effect in working memory tasks.

(Snowling, 2000, 37)

Everybody's working memory has a capacity limit and, due to the processing effort taken to decode letters, words and sentences, this is reportedly less in dyslexic learners (Snowling and Stackhouse, 2006). If Brady's (1991) pie analogy is used, dyslexic learners use up more of the available working memory "pie" in decoding verbal information, which then leaves less room for temporary storage of information. Hence, low working memory capacity affects the way information is processed: those with dyslexia process information differently from those 
without the condition and difficulty remembering audio and visual information can impair the ability to follow sets of instructions (Reid, 2007).

Working memory capacity may also have implications when dyslexic information users interact with new information found throughout a search and have to relate this information to the original search (SCONUL, 2011). Furthermore, in regards to the ability to evaluate sources, MacFarlane et al. (2012) found a link between working memory and the number of documents that were judged irrelevant in information retrieval tasks. It was found that a low working memory capacity impaired ability to judge documents as irrelevant (MacFarlane et al., 2012).

Long term memory stores information, such as vocabulary, spellings and facts previously learned (Vance and Mitchell, 2006). As words are stored in long term memory, dyslexics will have associated difficulties in retrieving the desired words, even if terms are familiar (Snowling, 2000). Furthermore, during information search tasks, long term memory will be constantly called upon to make sense of the information encountered in relation to previously held knowledge.

\subsection{Self-efficacy}

Bandura (1993) found that the higher the perceived self-efficacy of an individual, the higher the probability of the individual remaining task orientated, even when set-backs are encountered, and of them employing strong analytical thinking skills. It has been found that those with dyslexia have low self-efficacy in relation to educational tasks (Burden, 2008) and it is pertinent to investigate if these affective aspects also influence the ability to become information literate. Walton and Hepworth $(2011,468)$ found that "becoming information literate was associated with the affective state". In addition, Ford, Millar and Moss (2001) note that self-efficacy in information retrieval can be increased with intervention and this makes it an important area of study in user instruction for dyslexics.

\subsection{Assistive strategies}

Systematic approaches to the teaching and retention of new skills and information to dyslexic learners that have been shown to be most effective are the use of multi-sensory learning, including visual techniques, such as concept mapping, and overlearning (Reid, 2013; Stein and Saunders, 2012). Within the context of Higher Education, Pino and Mortari (2014) identified four key themes within students' personal coping strategies:

- $\quad$ study skills;

- compensatory strategies;

- help from family, friends and fellow students;

- meta-cognitive and meta-affective skills. 
People involved in the information literacy instruction of those with dyslexia must consider this holistic picture of how dyslexics are approaching their education. Meta-cognition and meta-affective skills allow students to consider their thinking and emotions as they progress through their learning, drawing on previous learning experiences. McLoughlin and Leather (2013) argue that these skills may not be developed in dyslexic students, as they may have had negative learning experiences in the past. Reid (2007) highlights the importance of tailoring support to the individual when providing study skills support and this sentiment can be applied to all instructional interactions with dyslexic learners.

Searching for and evaluating sources of information online involves interaction with a predominantly written medium and commonly employed coping strategies to help compensate for reading and writing difficulties include the use of assistive technology (Draffan, Evans and Blenkhorn, 2007) to read text aloud and to transfer speech to written text.

\section{Methodology}

If dyslexia presents barriers to learning, then it is a justifiable hypothesis that it will also present barriers to becoming information literate. In order to investigate this, a mixed methods approach was adopted, the research aim being to investigate the behaviours and thinking of the participants (Bryman, 2016) as they searched for sources of information for an assignment. Search behaviour was observed through the collection of screen recordings and participants' thinking about the search process was explored in interviews.

\subsection{Context}

The study was conducted in a small, alternative Higher Education provider which offers one BA (Hons) degree in Early Years Development and Learning. 98.5\% of students are female and in 2015-2016, 8\% of students had declared themselves as dyslexic. Students receive three information literacy instruction lectures in groups of around twenty in the first year of study. These sessions are titled 'Searching for information', 'Evaluating information' and 'Using information ethically'.

Emails calling for volunteers were sent at the beginning of the academic year in 2015 to second and third year students and in January 2016 to first year students. Participants were recruited from all three year groups and initial meetings arranged as soon after participants volunteered as possible, all within one week of this. The modules selected for inclusion had submission dates ranging from 24 January 2016 to 24 April 2016 and each participant came back in for an interview within two weeks of assignment submission.

\subsection{Protocol}

Data was collected in a three stage process; an initial meeting, screen recording diaries and a closing interview. 


\subsubsection{Initial Meeting}

There were five steps to the initial protocol; two questionnaires and three cognitive screening exercises. Background questions were asked, after which a fifteen question self-efficacy questionnaire was administered. This required participants to rate their efficacy from 0-100 in the three areas of:

- general academic work;

- searching using the internet;

- searching using Discovery, an online resource discovery database to which participants had access.

A score of 0 indicated they had no confidence in their ability and 100 meant they had complete confidence. A scale of 0-100 was chosen in alignment with Bandura's assertion that this provides more sensitive and reliable predictions of performance (Bandura, 2006).

The three cognitive screening exercises administered were; a digit span test, rapid automised naming and reading speed. A digit span test measures the phonological side of working memory, an area in which dyslexics show consistent and prevailing weakness (Snowling, 2000). Participants repeat increasingly long series of numbers, forward to begin with, then they are required to reverse the sequence and to continue until they have repeated two sequences incorrectly. Turner and Ridsdale's (2004) sequences were used. Rapid Automised Naming (RAN) was first developed as a cognitive measure in 1976 by Denckla and Rudel (1976) and it measures the ability to recall familiar words from long-term memory and shows information processing speed. Those with developmental dyslexia have been consistently shown to perform slower in these tasks in comparison to their peers (Norton and Wolf, 2011). Elliot and Grigorenko (2014) highlight that low scores in RAN indicate the ongoing difficulties of word retrieval for well-known vocabulary. The York Adult Assessment Battery-Revised (YAA-R) (Warmington, Stothard and Snowling, 2012), which recorded scores for one hundred and six university students with no history of reading difficulty, was used and participants were timed as they called out the names of familiar objects presented, in pictures, in lines. To calculate reading speed, a passage with one hundred and five words was presented on an iPad and the time taken to read aloud was recorded, noting errors, giving an indication of peer alignment with regards to reading capability.

\subsubsection{Screen Recordings}

If participants used a laptop with Windows, $B B$ Flashback Express (Blueberry Software Ltd., 2016) was downloaded and a demonstration given. If they had a Mac, instructions were given on how to use the Quicktime Player (Apple Inc., 2016). Participants were instructed to record all searches made for sources of information for one module and they were emailed and invited back for the closing interview once the module had been completed. 


\subsubsection{Closing Interview}

Participants submitted their reference list and were asked to reflect to what extent they felt their search for resources was successful, or otherwise, discussing successes and areas of difficulty.

\subsection{Ethical Considerations}

Approval was sought and granted from the Department of Computer Science Research Ethics Committee at City University, London. It was important that the study did not exert any undue pressures on the participants, as preparing for an assignment can already be deemed to be a stressful time. The interviews were carried out at the onset of modules and after the assignment had been completed, to minimise time spent in interviews during the academic semester and the time leading up to submission. It was encouraging to note that minimal disruption from having to stop and start the screen recording software was reported by participants and the use of Quicktime Player (Apple Inc, 2016) and BB Flashback (Blueberry Software Ltd., 2015) software was considered to be successful. The requirement to keep all data secure throughout the research process was accentuated by the sensitive nature of much of the data generated, in particular through the cognitive screening exercises. Furthermore, when viewing recordings for data analysis, anonymity was not always possible, as participants often had personal photographs as screen savers and it was common for pop-ups from Facebook and emails, which included names and usernames, to be visible. Of pertinence were occasions when participants signed in to platforms, such as Discovery and emails, when passwords were visible.

\subsection{Participants}

Eighteen participants were originally recruited, nine dyslexic and nine nondyslexic. Of the non-dyslexic group, one stated at the closing interview that she had decided to do her searches on an iPad and hence, had no recordings. Another returned an extremely low score in the digit span test, two percentile, and although she had previously been tested for dyslexia and this had not been diagnosed, it was decided that there may be another cognitive disability present and she was withdrawn from the study. Of the dyslexic group, one participant claimed to have forgotten to record any searches and another did not submit her assignment due to mitigating circumstances and was withdrawn from the study.

Results from fourteen participants are reported; seven dyslexic and seven nondyslexic (Table 1). Participants came from all three year groups and the modules they recorded searches for are shown in Table 2.All participants were female undergraduate students ranging in age from 18 (C8) to 27 (P7). The average age in the dyslexic group was 22 and for the non-dyslexic group this was 20 . 


\begin{tabular}{|l|l|}
\hline Dyslexic Participants & P1, P3, P5, P6, P7, P8, P9 \\
\hline Non-dyslexic Participants & C2, C4, C5, C6, C7, C8, C9 \\
\hline
\end{tabular}

Table 1: Participants.

\begin{tabular}{|c|c|}
\hline Module Title & Participants \\
\hline The Literature Review (Level 6) & C2, C5, C6, P1, P5, P7 \\
\hline Parenting Approaches (Level 5) & C7, C9, P3, P6, \\
\hline The Invention of Childhood (Level 4) & C4, C8, P8, P9 \\
\hline
\end{tabular}

Table 2: Participants' level of study.

\subsection{Screen Recordings Collected}

The total length of the recordings collected was fifteen hours, two minutes and twenty-two seconds. The non-dyslexic group submitted seven hours, forty-three minutes and fourteen seconds of recordings and the dyslexic group submitted seven hours, nineteen minutes and eight seconds. The least amount of time submitted by a participant was seven minutes forty-seven seconds (P7), which the participant estimated to be 5\% of total searches and the most was three hours, twenty-eight minutes and fifty-seven seconds (P8), which was stated to be $90 \%$ of total searches undertaken.

\section{Self-efficacy and Cognitive Screening Results}

To investigate cognitive and affective barriers to information literacy, the dyslexic participants' cognitive and affective profiles had to be analysed in comparison to their peers. Cognitive aspects were measured in the aforementioned cognitive screening exercises and efficacy was analysed through questioning. Participants were asked to rate their efficacy in fifteen skills (Table 3) and the average for the dyslexic and non-dyslexic group of participants are shown in Figure 1. 
4.1 Self-efficacy

\begin{tabular}{|l|l|l|l|}
\hline Q1 & I can plan university work & Q9 & $\begin{array}{l}\text { Discovery: I can find information inside } \\
\text { sources }\end{array}$ \\
\hline Q2 & I can organise university work & Q10 & Discovery: I can refine searches \\
\hline Q3 & $\begin{array}{l}\text { I can motivate myself to do } \\
\text { university work }\end{array}$ & Q11 & Internet: I can find appropriate sources \\
\hline Q4 & $\begin{array}{l}\text { I can find solutions to problems } \\
\text { assignment }\end{array}$ & Q12 & Internet: I can think of good keywords \\
\hline Q6 & $\begin{array}{l}\text { Discovery: I can find appropriate } \\
\text { sources }\end{array}$ & Q14 & Internet: I can find information inside sources \\
\hline Q7 & $\begin{array}{l}\text { Discovery: I can think of good } \\
\text { keywords }\end{array}$ & Q15 & Internet: I can follow links to expand searches \\
\hline Discovery: I can evaluate sources & Internaluate sources \\
\hline
\end{tabular}

Table 3: Self-efficacy questions asked.

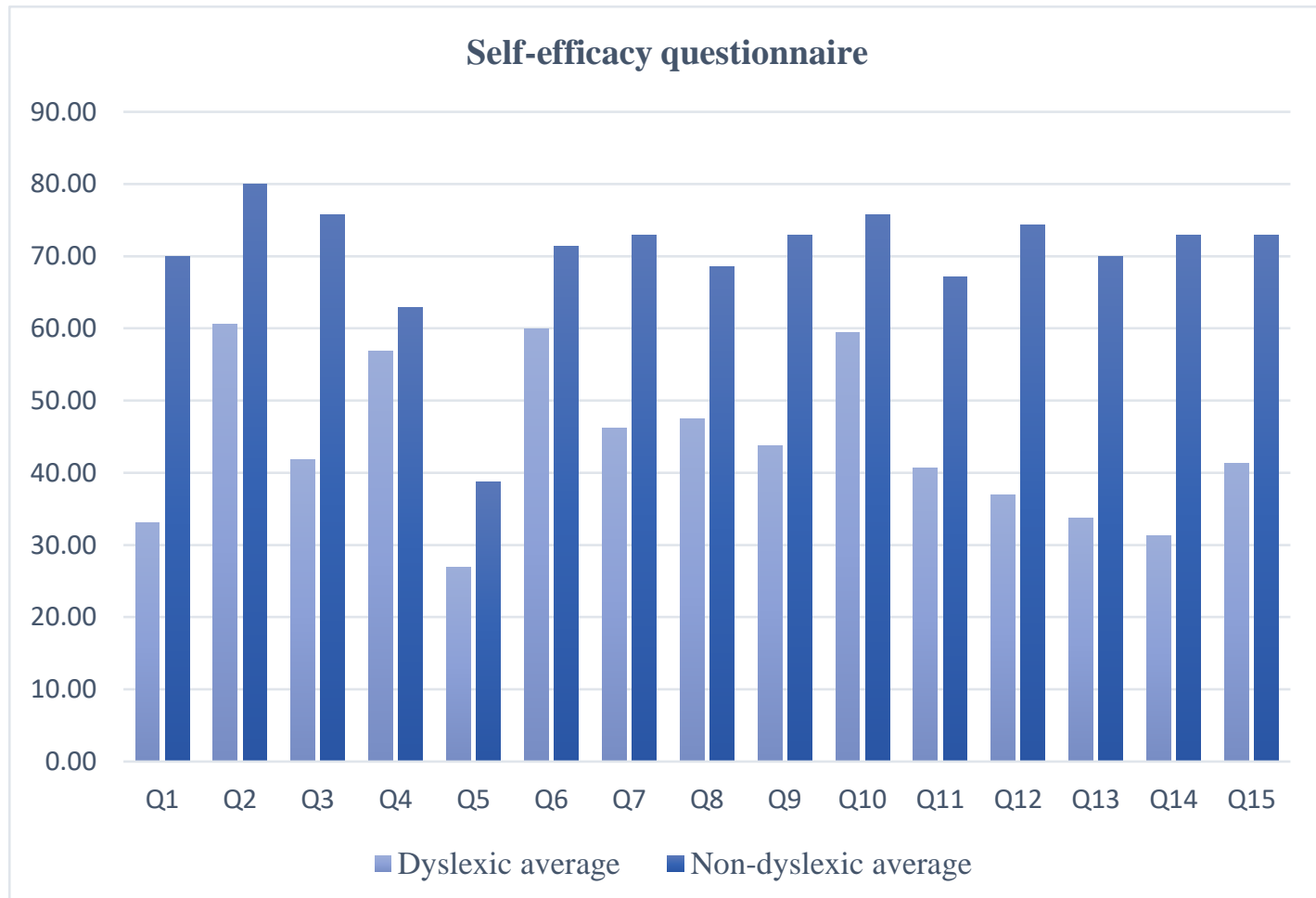

Figure 1: Average scores for each group given in response to questions in Table 3. 
Dyslexic participants rated their ability in every skill as lower than the nondyslexic group. The most significant discrepancy was in the dyslexic groups' efficacy in their ability to find information from inside sources located on the internet, with a 42.5 point difference in the average the groups. The difference was still notable for locating information inside sources found in Discovery, but there was slightly less difference than for internet sources, at 31.25 points. This suggests that even if searches can be carried out successfully, there are still considerable difficulties extracting the necessary information from sources found.

The dyslexic group rated their efficacy in the evaluation of internet sources as very low across all levels of study, which implies that this is a skill that does not improve with increased experience. Planning returned the next biggest difference in efficacy, and it can be surmised that this lack of ability to plan academic work will have a detrimental effect on the planning of searches. This low score was closely followed by the ability to generate good keywords when using the internet. Efficacy in the ability to generate keywords when using Discovery was slightly higher, but the disparity between the ratings of the two groups was still considerable, at 28.75 points. The lower scores when searching on the internet suggest that there needs to be more focus on skills needed to navigate the web in the initial information literacy training for dyslexic learners.

\subsection{Working Memory}

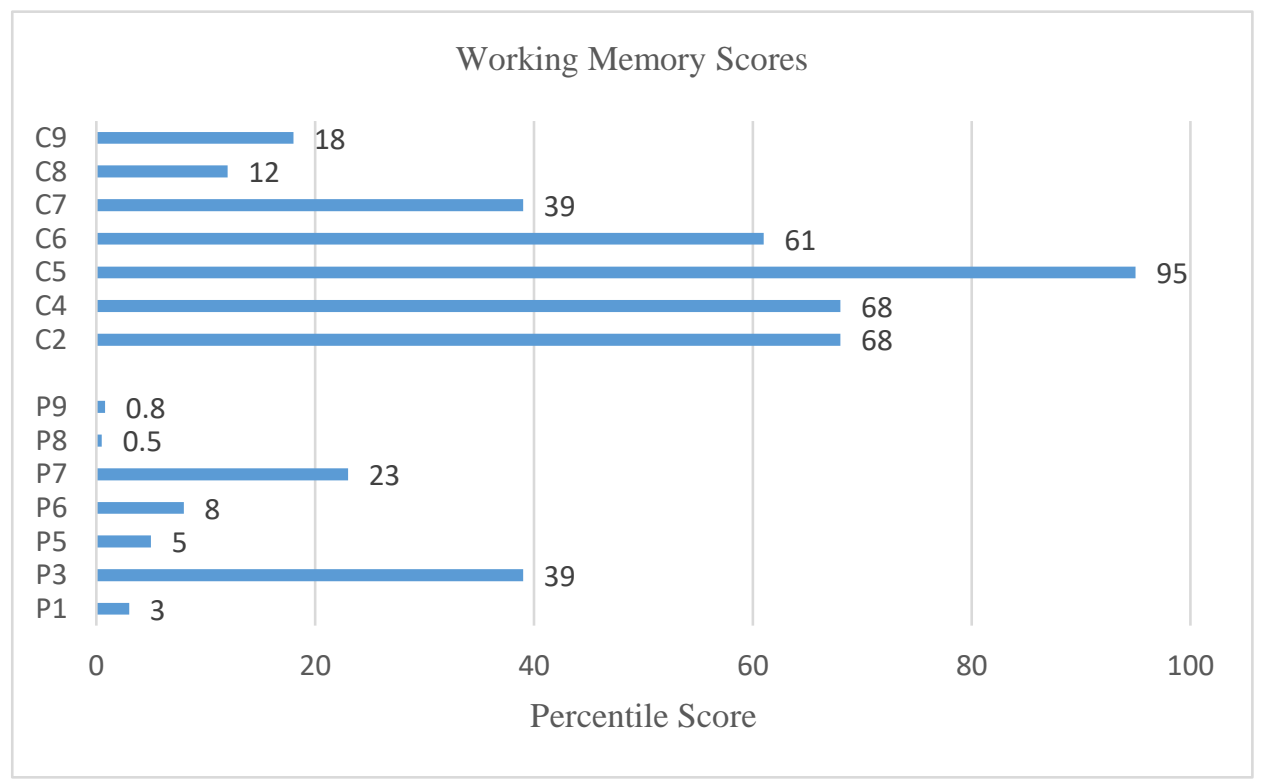

\section{Figure 2: Working Memory Scores.}

The percentile score refers to how the individual performs in relation to the rest of the population. If a person is in the 1 percentile, $99 \%$ of the population would perform better. Two non-dyslexic participants returned relatively low scores for working memory $(\mathrm{C} 8, \mathrm{C} 9)$, but both scored above average for reading speed, indicating that there is no reading impairment present. Figure (2) shows that, as would be expected, the majority of the dyslexic group's working memory scores are significantly lower than their peers. 
The YAA-R (Warmington, Stothard and Snowling, 2012) mean is 1.88 words per second for RAN and 2.73 words per second for reading, and these rates can be used to give an indication of how the participants compare to their peers.

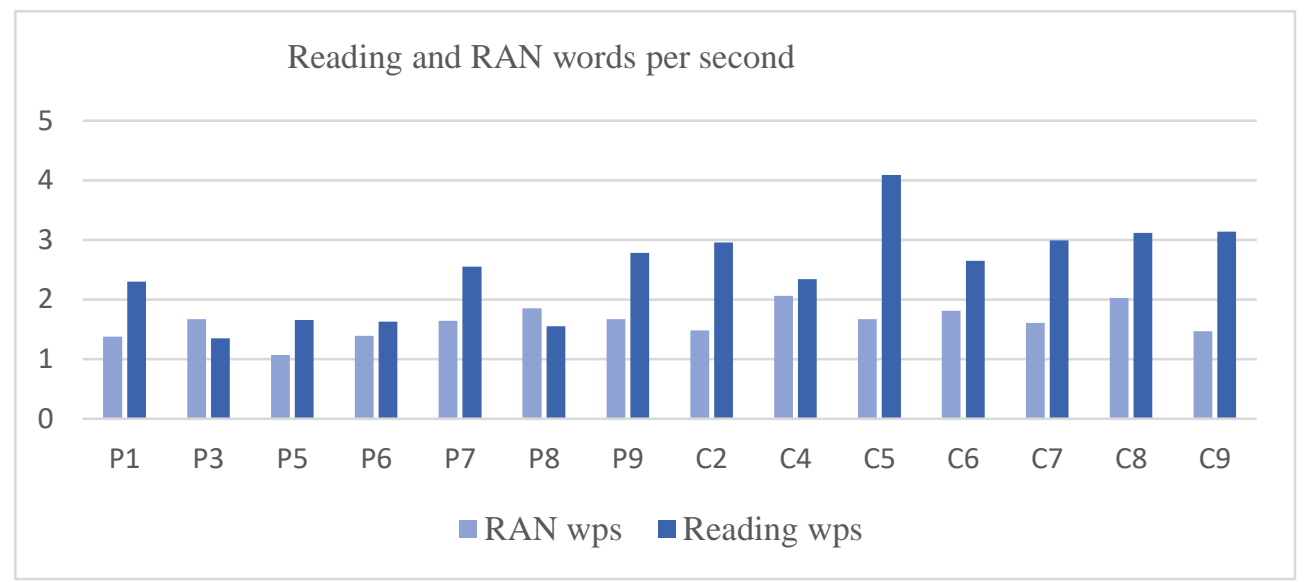

Figure 3: RAN and Reading words per second.

The dyslexic group, with the exception of P9, were reading at a below average rate. Two of the non-dyslexic participants were also reading slightly slower than average. These results are indicative of adults accessing Higher Education.

In the rapid naming exercise, one non-dyslexic participant (C9) and three dyslexic participants (P1, P5, P6) were more than half a word per second below the YAA$\mathrm{R}$ mean, indicating difficulties in the recalling of familiar words from long-term memory.

\section{Data Analysis}

The pillars within the model of information literacy offered by SCONUL (2011) are used here to analyse the data. The interview answers give insight into the attitudes and behaviours (understanding) necessary to be information literate and the screen recordings show where skills and competencies (ability) are demonstrated. Participants discussed the searches they conducted on the World Wide Web and in Discovery, an online database used in the setting.

5.1 SCONUL Pillar elements: Identify: identify a search topic and define it using simple terminology; Plan: revise keywords according to the resources available and / or results found

\subsubsection{Keywords}

All participants were observed to be able to outline a search question "using simple terminology" (SCONUL, 2011, 5), but three of the dyslexic group (P1, P3, P9) discussed difficulty in generating effective search terms for their topics. P1 and P3 stated: 
I don't think Discovery knew what I meant... you've got to do the proper wording... and kinda be more accurate.

I still think I struggle with using, finding the keywords that need to go in to make it [Discovery] work

These comments were in contrast to $\mathrm{C} 4$, who when asked "what went well?" with her searches, replied, "I think the words that I chose to search". Furthermore, there were difficulties stated and observed within the dyslexic group's behaviours when it came to revising keywords. Rather than generate new search terms from knowledge or in relation to the results returned, P1 relied on features of the search database to do this, commenting that she found the 'subject terms' offered in Discovery useful, as it gave alternate ways to word key terms. P1 gave the example, "you've typed in fathers and it's got fatherhood" (P1). A similar process was outlined by $\mathrm{P} 9$ who, whilst referring to a list of e-book titles that had been circulated to her, stated that:

that was quite useful as well, 'cause it gave you basic wording, like 'early childhood education'. I'd never thought to type that into Discovery [and that this then] triggered other things.

In the observations of P9's keyword search terms used, she was seen to use the phrase "world war 2" written in numerical form six times, in six separate searches. This was added to other search terms, such "education" and "children", but no alternative phrasing of the key search term was considered. These observations reinforce her cautiousness when it comes to thinking of alternative ways to phrase search terms. A similar pattern was observed in the screen recordings of P6, where she is seen using the search phrase "parenting styles" for a search relating to a module entitled Parenting Approaches in three separate searches, with no alternative terms observed or stated.

Difficulties in the revision of keywords were underpinned in several incidences by challenges in identifying concepts. This was defined as the capacity to break down larger topic headings into more specific keywords by three of the dyslexic group. P5 stated that, searching "was easier for more general things" and that she had difficulty "trying to find specific things". P8 and P9 reported similar problems in "the whole breaking the big search down to just the things I needed" (P8) and the fact that "you have to be quite specific about what you type in" (P9).

Two of the non-dyslexic group reported the ability to break down larger topics into smaller concepts and follow these in the search as a strength. C8 described success in "tracking down [the] specific", and in "going down and down and 
down" in her searches. This was also discussed by $\mathrm{C} 2$, who stated "it's just how you word things sometimes" and that she:

was going to talk about the history of technology, but it was too complicated, so then I just focussed on how it has become more part of society.

One key difference between the groups was that five of the non-dyslexic group spoke of and demonstrated the ability to adapt the search terms used to new topics as they were encountered in the search process. As evidenced above, this understanding was not stated or observed in the dyslexic group. C4, C5, C6, C8 and C9 spoke of or demonstrated the ability to use new knowledge and phrases as their searches progressed, incorporating new information into their search tactics. This manifested itself in searches where C5, C6 and C8 were observed using new phrases which they had encountered when reading web documents to perform new searches. Similarly, C4 and C9 were observed using key phrases from lecture PowerPoints for some of their searches and C9 stated:

I search main topic headings and then if something is introduced in the article and then follow this up and go more in depth.

P7 was observed extending her searches by using an 'articles citing this article' option, but she was the only dyslexic participant to expand searches in this way in the recordings collected for this study.

\subsubsection{Suggested Search Terms}

There was considerable difference in the utilisation of drop-down keyword suggestions and alternate search term options used between the two groups and it is surmised that these tactics were used to compensate for the challenges the dyslexic group faced in generating and revising keywords.

P3, P5, P6, P8 and P9 were all observed to use drop down suggested search terms frequently, on all platforms used. P8 clicked on "Search instead for" and "showing results for" features, even when this meant that the selection was either very similar to the original search wording, or changed the meaning of the search completely. Examples included:

- Search [1]: Original search "ethelred the unready ruling on wife bearing child" > 'Search instead for "ethelred the unready ruling on wife beating child".

- Search [2] original search, "ways in which children were effected by the black death", clicked 'Showing results for' "ways in which children were affected by the black death".

- Search [10] original search "18th century childhood" > clicked related search "children in the $18^{\text {th }}$ century" > clicked related search " $18^{\text {th }}$ century childhood". 
These observations were in contrast to the non-dyslexic group, where three of the non-dyslexic group used only one drop-down suggestion $(\mathrm{C} 4, \mathrm{C} 5, \mathrm{C} 7)$ in their recordings and two others used this feature four times $(\mathrm{C} 8, \mathrm{C} 9)$.

\subsubsection{Vocabulary and Spelling}

In addition to the generation and revision of search terms, the spelling of these words hindered some in the dyslexic group. There were three cases where the misspelling of words had a detrimental impact on the search. P5 had considerable impediment spelling the word "behaviour", misspelling this eight times over three separate searches (Search $[1,2,3])$. On one occasion the solution implemented was to copy and paste the word from another document. When no or few results were returned due to spelling, one time as "behavuour", the "search instead for", "results may also be available" and "did you mean" features of Google were selected. This resulted in the American spelling, "behavior" being used several times. P8 misspelled the name "McMillan" as "McMillian" in four separate searches (Search $[5,14,17,18])$ and followed her tactic outlined above of using the Google suggested search terms. P9 made several spelling errors, including typing "echological" for "ecological" (Search [5]) and "defintion" for "definition" (Search [4]). P9 used the drop down and "did you mean" suggestions as a solution and searched using these words instead of the ones originally typed.

All seven of the non-dyslexic group were observed to make typing errors when entering search terms. These were minor errors and were all remedied directly before the search was begun and hence did not have an impact on the search results returned.

In addition to difficulties with the spelling of terms, new vocabulary caused two dyslexic participants to pause their search and find definitions of words used, before they could make a decision as to whether the source was appropriate for their needs. When new vocabulary was encountered, two of the dyslexic group used Google to define words. P3 searched for a definition of "pre-emptive" and P8 searched for the definition of "pauper", clicking on the "Read Aloud" option in Google for this word.

\subsection{SCONUL Pillar elements: Plan: construct strategies for locating information; Gather: use a range of retrieval tools}

\subsubsection{Refining and Expanding Searches}

A range of retrieval tools were observed being used by both groups in the screen recordings, with varying success. Tactics to refine and expand searches observed among the non-dyslexic group (C2, C4, C5, C7, C8) included the use of filters and Boolean Operators. Only C5 and C8 were observed using retrieval tools to expand the number of results returned: Uniquely, C5 was observed using the OR Boolean function to expand one search (Figure 4), demonstrating an understanding that "complex search strategies can make a difference to the breadth and depth of information found" (SCONUL, 2011, 7). C8 used "child*" as a search term to expand searches and a language filter when results returned were in languages 
apart from English. C8 also used the publication date and source type filter, showing an awareness of several of the tools available to her. Similarly, C5 used filters in several searches. C4 and C5 used the geography filter when searching for information about British childhood, showing the ability to consider appropriate methods to refine searches when searching for context-specific information.

\begin{tabular}{l} 
Searching: Discovery Service for Norland College \\
\begin{tabular}{|l|l|}
\hline family services & Select a Field (option... \\
\hline Children centres & Select a Field (option... \\
\hline Basic Search Advanced Search Search History
\end{tabular} \\
\hline
\end{tabular}

Zesults

Search Results: 1 - 20 of 2,582,535

Figure 4: screen shot from C5 search [1].

\subsection{SCONUL Pillar element: Gather: construct complex searches}

\subsubsection{Constructing complex searches}

In the dyslexic group, the ability to "construct complex searches" (SCONUL, $2011,8)$ proved problematic, as several search strategies were employed that proved to be ineffective. In interview, P3 reported difficulty in removing irrelevant results, the "things that were extraneous that I didn't need" and these challenges were highlighted in search [2], where P3 was observed to use several Boolean Operators (Figure 5), reporting that she, "found nothing useful".

\section{New Search Publications myday Moodle}

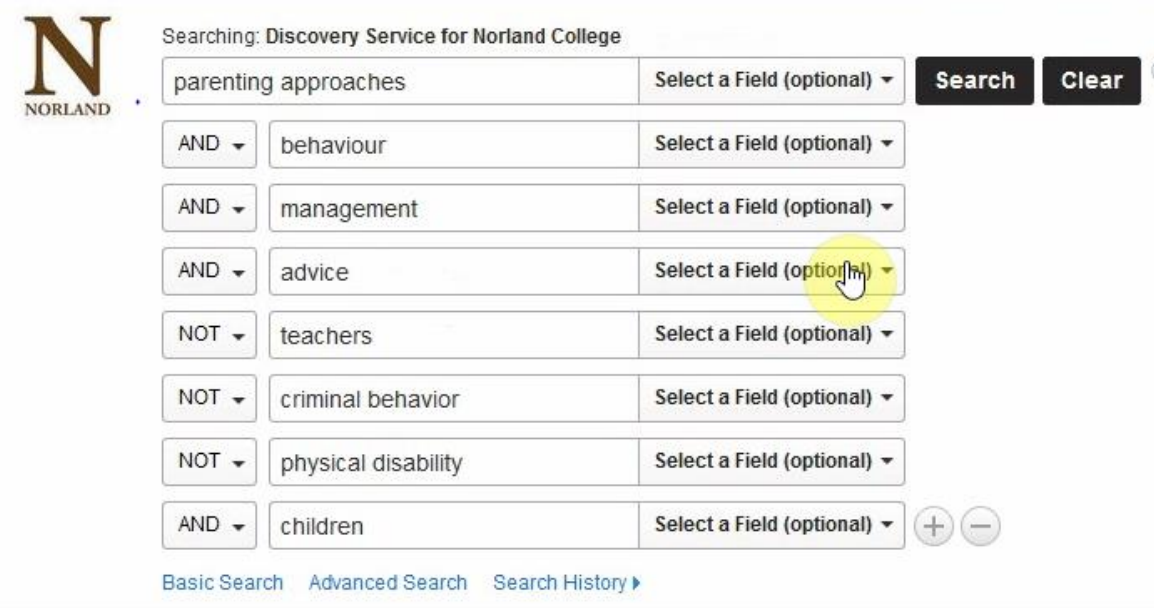

Refine Results

Search Results: 1 - 20 of 4,140

Figure 5: screen shot from P3 search [2]. 
P6 was also observed to use filters and Boolean Operators heavily in her searching, this was combined with the use of quotation marks in one search that returned only five results (Figure 6) and can be deemed to be ineffective.

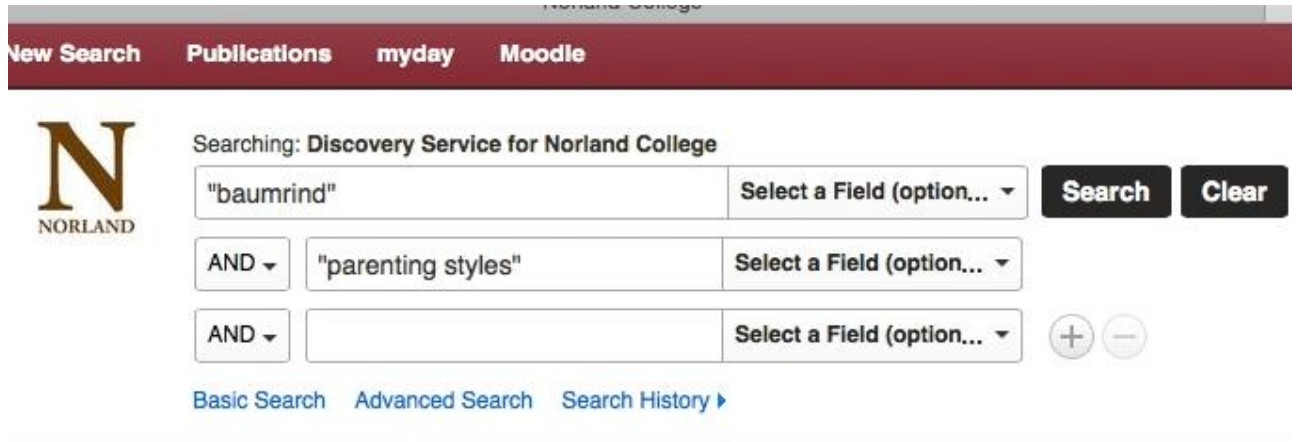

Refine Results

Search Results: 1 - 5 of 5

Figure 6: screen shot from P6 search [5].

P8 faced considerable difficulties refining searches, stating that when trying to use Discovery she "forgot everything". She detailed the bits she found confusing as,

when you go to advanced search to then try and only pick the bits that I wanted and not all the bits... I just gave up on Discovery [as the Boolean Operators] didn't make any sense.

P8 can be observed utilising filters in only one of 22 search sessions, (Search [12]). A date filter was applied, which only returned searches from June 2002. This search is then temporarily abandoned, before returning and applying a source type filter and clicking on a source. In contrast, successes were observed for P1, who was seen to use a selection of source type, geography and language filters to help refine searches.

These observations suggest that the dyslexic group are using filters and Boolean Operators to try and reduce the number of results that they have, while the nondyslexic group are more comfortable expanding the results returned.

\subsection{SCONUL Pillar element: Gather: locate and access information, access full- text}

\subsubsection{Search within sources}

The ability to access full-text information (SCONUL, 2011) was evident throughout the searches of both groups; however, there was an observed difference in features utilised to find information from inside sources. When navigating within sources, the "Table of contents" feature was used by three dyslexic participants and six non-dyslexic (P3, P7, P9, C2, C4, C5, C7, C8, C9). Two other tools were observed being used, "CTRL + F" (P6, P9, C2, C5, C6, C9) 
and the "Search within" feature offered in some websites and Discovery (P8, P9, C4, C6). C7 also used the "Most relevant Pages" feature in Discovery. During the recorded searches, all non-dyslexic participants used one or more of these tactics to locate information inside sources; this was only the case for three of the dyslexic group (P6, P8, P9). When this is considered in relation to the low selfefficacy scores returned by the dyslexic group, it gives an indication that the nondyslexic group are far more confident and able to locate the information they require once a source has been selected.

\subsection{SCONUL Pillar element: Evaluate: assess the quality of information and relate the information found to the original search strategy}

\subsubsection{Evaluating Sources}

Every participant when asked, "overall, are you happy with the sources found?" responded in a positive manner, but tactics used varied. Saving sources in quick succession to then come back and evaluate usefulness was a tactic employed by $\mathrm{P} 1$ and $\mathrm{C} 2$. In order to evaluate sources, P1 reported in the initial interview that she would print documents and then decide which ones to use, and this strategy was evident in the recordings. During a search session lasting thirty one minutes, thirteen documents were printed, of which only one was used in her assignment. The sequence followed for each document selected was "Add to Folder" > "PDF Fulltext" > "Print". Furthermore, there was confusion evident in the organisation of sources by P1 as, of the folders viewed within search [1], two had the same words as the title, "shared parental leave" and "parental leave shared". P1 was observed saving documents to both folders during the same search. C2 followed a similar tactic; in a twelve minute search, she "opened in a new tab" and "saved to folder" nine sources, two of which were eventually used. In the interview, C2 stated that she was aware of need to look for author and date in internet sources, showing understanding of the need to assess the quality of sources (SCONUL, 2011) and perhaps contributing to finding more useful sources in a quicker time than P1. In regards to organisation, C2 arranged sources found in folders by source type, giving them a title based on content, showing the ability to summarise content quickly. She said in interview that she would group them by topic next time.

When discussing the evaluation of sources, P6 stated, "I found one I just thought this is totally irrelevant, I don't even understand what it is saying", suggesting that she may have been accessing unsuitable content for her level of study. Similarly, P8 had a low self-efficacy for evaluation of sources and was observed using commercial websites when on the internet. In discussions about evaluating sources, she claimed that she felt it was more about "evaluating what I knew" (P8).

When reading to judge for relevance, two dyslexic participants were observed to use assistive technologies; P3 used Read and Write (Texthelp, 2015) once to read an abstract and P6 used Claroread (Claro Software, 2015) for this, but this use was minimal, suggesting that the dyslexic group were not utilising technological assistance for their searches. 
There was a confidence exhibited in the non-dyslexic group with regards to accessing more sources than necessary and then having the ability to select appropriate content. C7 acknowledged that it could take "long time to find a source" as she would read a lot and then find that the information was not relevant:

you'd find an article, you'd read and you'd go, actually that's not really relevant, it'd take a long time to find a source and think 'yes, it's good' and then write about it.

C7 stated that she thought it was good that there was "lots to choose from" and after deciding which ones were relevant she was "happy with them all". C8 mirrored this view, stating "yeah I think I found some good stuff". C6 and C5 spoke of the fact that they found too many sources and too much information: "I had too much, I had to cut lots of bits out" (C6), "a lot of the reading I did didn't end up going in" (C5). C4 highlighted that evaluating sources took time and thought, but that she was aware that some sources were not appropriate to use,

\section{I did get a bit confused with sites that probably weren't appropriate and then I'd} have to take them out from my bibliography.

C4 also showed an awareness of the need to evaluate sources when she stated, "I used Discovery mainly, just because I wanted to keep the searches safe".

The fact that the non-dyslexic participants reported that they read a lot more information than was actually finally used, something that was not described by the dyslexic group, could imply that the non-dyslexic group are more comfortable taking longer to search and read more content than the dyslexic group. The nondyslexic group acknowledged that it is an extended process required to make informed decisions as to what information they require, a requisite to the ability to "relate... information found to the original search strategy" (SCONUL, 2011, 9), something that was not evident in the dyslexic group.

\section{Implications for Practice}

P1, P5 and P6 had low scores for the recall of words from long-term memory and all displayed difficulty in the creation of keywords. This manifested itself in the inability to consider alternate phrasing of familiar terms. Furthermore, there was no evidence observed suggestive that the dyslexic group were able to adapt their search terms as searches continued, with a heavy reliance on suggested search terms by the whole group. Concept mapping, something that is used by dyslexics as a study skill strategy (Reid, 2013), is suggested to assist in the generation of key words and phrases before the search has begun and then again throughout the search as new information is encountered. This will allow new knowledge to be incorporated into search strategies and to develop understanding of how the phrasing of search terms can impact on results returned.

Challenges faced in the utilisation of appropriate retrieval tools that will extend searches, rather than narrow results to ineffective numbers, require further 
investigation. It is necessary to ascertain whether the tactics observed are a result of working memory capacity and consider how these difficulties can be overcome in the search process. Following a complex search strategy is similar to following a set of instructions, which dyslexics have been show to demonstrate difficulty in doing, due to working memory restrictions. An overload on working memory would explain why the dyslexic information users felt it necessary to narrow searches to such an extent. When delivering information literacy training, trainers should bear in mind effects on working memory load.

The low self-efficacy scores recorded for the dyslexic group are of real concern: if a person does not believe in their ability in search tasks, the likelihood of them persevering when difficulties are encountered is low. Efficacy is an area that those working with people with dyslexia must take into consideration in interactions and overlearning, as well as meta-affective approaches - which students are already applying to learning (Pino and Mortari, 2014) - should be employed during information literacy instruction. Subsequently, if skills are taught and then reinforced over a long period of time, and dyslexics are asked to consider their awareness of how they feel at different stages in the training, this will allow dyslexic learners the opportunity to increase efficacy in personal ability and, hence, increase the likelihood of prolonged and more productive searches. The low self-efficacy within the dyslexic group in their ability to locate information from inside sources and in the evaluation of internet sources highlight these areas as key components of information literacy for overlearning. It would appear that there is only minimal use of assistive technologies used to help in the evaluation of sources and their under-use may be indicative that they are not considered useful by students when searching for sources.

\section{Limitations of the study}

The study has been undertaken in a Higher Education College, and therefore cannot be deemed fully representative of the wider dyslexic population. As all participants were female, this has provided a gender skewed sample and future studies will endeavour to recruit a balanced gender split in participants to address this. There were a couple of hardware issues which reduced the amount of recordings taken by two participants: as the screen recording files required a large amount of available storage space, one participant's (P7) ability to record searches was hindered by the lack of memory storage space available on her laptop and one recording submitted by C6 was a corrupted file and could not be viewed.

A limitation of the self-efficacy questionnaire was that it did not allow the reasons behind these low scores to be investigated, by asking why participants felt that they could not complete these tasks. Future research will seek to ask these additional questions.

\section{Conclusion}

The differences in search strategies between the two groups suggests that dyslexia does have an impact upon the skills required to become information literate and it will be pertinent in the future to build up a fuller understanding of this profile of 
information users. Common difficulties reported and observed within the dyslexic group manifested themselves in: the creation, adaptation and spelling of keywords; the ability to utilise appropriate strategies for the expansion and refining of searches; and self-efficacy in the skills needed to search for and evaluate sources. The impact of working and long-term memory will be key factors in future studies, and ways in which information overload for those with dyslexia can be tackled within searches for information require further consideration within information studies.

It will be important in the future to extend these investigations into cognitive and affective aspects and their influence to include all areas of SCONUL's and other models, and to those with dyslexia who are not accessing Higher Education, as this will ensure that theories drawn are relevant to the wider population.Although ultimately it must be remembered that every dyslexic individual has a unique cognitive profile and instruction must be tailored as such, further research will help to identify common characteristics in dyslexic information users that can be used to guide information literacy instruction and support.

\section{References}

ACRL (2015) Framework for Information Literacy for Higher Education. URL: http://www.ala.org/acrl/standards/ilframework [accessed 25.07.2015].

ALA (2015) Information Literacy Competency Standards for Higher Education. URL: http://www.ala.org/acrl/standards/informationliteracycompetency [accessed 29.08.2015].

Apple Inc. (2016) QuickTime Player. URL: http://www.apple.com/uk/quicktime/ [accessed 21.01.2016].

Bandura, A. (1993) Perceived Self-Efficacy in Cognitive Development and Functioning. Educational Psychologist, 28(2), 117-148.

Bandura, A. (2006) Guide for Constructing Self-Efficacy Scales. In: Pajares, F., and Urdan, T. (eds.) Self-Efficacy Beliefs of Adolescents. Greenwich: Information Age Publishing. 307-337.

Berget, G., Mulvey, F. and Sandnes, F.E. (2016) Is visual content in textual search interfaces beneficial to dyslexic users? International Journal of Human Computer Studies, 92-93, 17-29.

Blueberry Software Ltd. (2016) BB Flashback Express Download. URL: http://www.bbsoftware.co.uk/BBFlashBack_FreePlayer.aspx [accessed: 21.01.2016].

Brady, S. A. (1991) The role of working memory in reading disability. In: Brady, S.A. and Shankweiler, D. (eds.) Phonological Processes in Literacy. 129-151. 
British Dyslexia Association (2013) Dyslexia and Specific Learning Difficulties in Adults. URL: http://www.bdadyslexia.org.uk/dyslexic/dyslexia-and-specificlearning-difficulties-in-adults [accessed 23.12.2015].

Bryman, A. (2016) Social Research Methods. $5^{\text {th }}$ ed. Oxford: Oxford University Press.

Burden, R. (2008) Is dyslexia necessarily associated with negative feelings of self-worth? A review and implications for future research. Dyslexia, 14(3), 188196.

CILIP (2013) Information Literacy Skills. URL:

http://www.cilip.org.uk/sites/default/files/documents/Information\%20literacy\%20 skills.pdf [accessed 02.01.2016].

Claro Software (2015) Claro. URL: https://www.clarosoftware.com [accessed 27.06.2016].

Denckla, M.B. and Rudel R.G. (1976) Rapid automatized naming (R.A.N): dyslexia differentiated from other learning disabilities. Neuropsychologia, 14(4), 471-79.

Draffan, E., Evans, D. and Blenkhorn, P. (2007) Use of assistive technology by students with dyslexia in post-secondary education. Disability \& Rehabilitation: Assistive Technology, 2(2), 105-116.

Elliott, J.G. and Grigorenko, E.L. (2014) The Dyslexia Debate. London: Cambridge University Press.

Ford, N., Miller, D. and Moss, N. (2001) The Role of Individual Differences in Internet Searching: An Empirical Study. Journal of the American Society for Information Science and Technology, 52(12), 1048-1066.

Higher Education Statistics Agency (HESA) (2015) HESA Free Online Statistics. URL: https://www.hesa.ac.uk/free-statistics [accessed 02/01/2016].

MacFarlane, A., Albrair, A., Marshall, C. R. and Buchanan, G. (2012) Phonological working memory impacts on information searching: An investigation of dyslexia. In: Kamps, J., Kraaij, W. and Fuhr, N. (eds.) Proceedings of the 4th Information Interaction in Context Symposium: Nijmegen, the Netherlands, August 21-24, 2012. New York: ACM. 27-34.

MacKenzie, I.S. (2013) Human Computer Interaction: An Empirical Research Perspective. Waltham, MA: Morgan Kaufmann.

McLoughlin, D. and Leather, C. (2013) The Dyslexic Adult: Interventions and Outcomes - an Evidenced-based Approach. Oxford: John Wiley and Sons. 
Norton, E.S. and Wolf, M. (2011) Rapid Automatized Naming (RAN) and Reading Fluency: Implications for Understanding and Treatment of Reading Disabilities. The Annual Review of Psychology, 63, 427-452.

Pino, M. and Mortari, L. (2014) The Inclusion of Students with Dyslexia in Higher Education: A Systematic Review Using Narrative Synthesis. Dyslexia, 20(4), 346-369.

Reid, G. (2007) Dyslexia. London: Continuum Books.

Reid, G. (2013) Dyslexia and inclusion: Classroom approaches for assessment, teaching and learning. 2nd ed. Oxford: Routledge.

Rose, J. (2009) Identifying and Teaching Children and Young People with Dyslexia and Literacy Difficulties. URL:

http://webarchive.nationalarchives.gov.uk/20130401151715/http://www.education .gov.uk/publications/eOrderingDownload/00659-2009DOM-EN.pdf [accessed 31.12.2015].

SCONUL (2011) The SCONUL Seven Pillars of Information Literacy. Core Model for Higher Education. URL:

http://www.sconul.ac.uk/sites/default/files/documents/coremodel.pdf [accessed 02.01.2016]

Smythe, I. (2011) Dyslexia. British Journal of Hospital Medicine, 72(1), 39-43.

Snowling, M. (2000) Dyslexia. Oxford: Blackwell.

Snowling, M. and Stackhouse, J. (eds.) (2006) Dyslexia, Speech and Language: a practitioner's handbook. London: Whurr.

Stanovich, K. E. and Siegel, L. S. (1994). Phenotypic performance profile of children with reading disabilities: A regression-based test of the phonologicalcore variable-difference model. Journal of Educational Psychology, 86, 24-5.

Stein, J. and Saunders, K. (2012) Dyslexia Handbook. UK: British Dyslexia Association.

Texthelp (2015) Read and Write. URL: https://www.texthelp.com/engb/products/read-and-write-family [accessed 27.06.2016].

Turner, M. and Ridsdale, J. (2004) The Digit Memory Test. URL: http://www.dyslexiainternational.org/content/Informal\%20tests/Digitspan.pdf [accessed 25.06.2016].

Vance, M. and Mitchell, J.E. (2006) Short-term memory: assessment and intervention. In: Snowling, M. and Stackhouse, J. (eds.) Dyslexia, Speech and Language: a practitioner's handbook. London: Whurr. 143-166. 
Walton, G. and Hepworth, M. (2011) A longitudinal study of changes in learners' cognitive states during and following an information literacy teaching intervention. Journal of Documentation, 67(3), 449-479.

Warmington, M., Stothard, S.E. and Snowling, M.J. (2013) Assessing dyslexia in higher education: the York adult assessment battery-revised. Journal of Research in Special Education Needs, 13(1), 48-56.

\section{Open access and copyright}

Library and Information Research is an open access journal. A freely available copy of this paper may be downloaded from the journal's website: http://www.lirgjournal.org.uk.

Copyright and associated moral rights in works published in Library and Information Research are retained by the author(s) but this paper may be used freely, with proper attribution, in educational and other non-commercial settings. 